\title{
Bacteriological Profile and Antibiogram of Catheter Related Blood Stream Infections (CR-BSIs) among Chronic Hemodialysis Patients Attending Tertiary Care Medical College Hospital
}

\author{
S. Karthiga ${ }^{1}$, A. Dhanasekaran ${ }^{2}$ and R. Someshwaran ${ }^{2 *}$ \\ ${ }^{1}$ Karpagam Faculty of Medical Sciences and Research, Othakkalmandapam, \\ Coimbatore-641032, Tamil Nadu, India \\ ${ }^{2}$ Department of Microbiology, Karpagam Faculty of Medical Sciences and Research, \\ Othakkalmandapam, Coimbatore-641032, Tamil Nadu, India \\ *Corresponding author
}

Keywords

Chronic kidney disease, Septicemia, Bacteremia, Biofilm, Infection

control, Antibiotic policy

Article Info

Accepted:

10 October 2018

Available Online:

10 November 2018

\section{A B S T R A C T}

Central Venous Catheter (CVC) remains a suitable form of vascular access for patients with chronic hemodialysis and has been estimated that almost $30-40 \%$ of patients are dependent on CVCs for their vascular access. Aim of the study is to determine the prevalence of Catheter Related Blood Stream Infections (CRBSIs). A Cross sectional study was undertaken after ethical committee approval, Blood samples $(5 \mathrm{ml}-10 \mathrm{ml})$ were collected from catheter site under sterile precautions and Blood Culture (Bac' $\mathrm{T}$ 'Alert) performed and isolates grown were identified by routine methods as per standards. Antibiotic Susceptibility Testing was also performed and results noted. Out of the 60 samples processed 24 samples $(40 \%)$ were "Culture Positives" and 36 samples $(60 \%)$ were sterile and termed "Culture Negatives" after 5 days of incubation. The predominant Gram positive organism observed in our study was MRCONS followed by MSCONS. The prevalence of CRBSIs among chronic hemodialysis patients with central venous catheter in- situ was found to be $40 \%$. Improving basic infection control measures and increasing the awareness by educating the health care providers could significantly reduce the occurrence of bacteremia could possibly aid in early diagnosis.

\section{Introduction}

Catheter Related Blood Stream Infections (CRBSIs) refers to bloodstream infection attributed to an intravascular catheter by quantitative culture of the catheter tip or by the difference in growth between catheter and peripheral venipuncture blood culture specimens (Harshal Shah et al., 2013).
Traditional classification of blood stream infections constitutes two variants viz., a. Community acquired, b. Health care associated or Hospital- acquired or Nosocomial infections (Friedman et al., 2002). Central Venous Catheter (CVC) remains a suitable form of vascular access for patients with chronic hemodialysis and has been estimated that almost $30-40 \%$ of patients with 
chronic hemodialysis are dependent on Central Venous Catheters (CVCs) for their vascular access. Nosocomial infections are the major cause for repeated hospitalization and death in hemodialysis patients in many countries (Masashi Suzuki et al., 2016). The prevalence of Catheter Related Blood Stream Infections ranges between $10-30 \%$ in hemodialysis patients which occurs at a rate of 0.5-47/1000 catheter days (Ramasubramanian et al., 2014). Catheter Related Infections are associated with substantial morbidity and mortality. Therefore, it is a potential menace and clinical challenge for Nephrologists and Physicians to diagnose CRBSIs early and to initiate appropriate management to prevent further complications. Strict adherence to Catheter care and Universal Safety Precautions by the health care providers will reduce the incidence of CRBSIs to a significant level.

Catheter Related Infections results from migration of skin commensals along the catheter into the blood stream or by contamination and or by colonization of catheter lumens by pathogenic microbes. Prevention strategies are directed at decreasing the growth and adherence of pathogens to catheter hub and or to the surface (Abhilash Chandra et al., 2016). Recently, promising techniques like Antibiotic Lock Therapy was introduced where antimicrobial solutions are instilled into the lumen of the catheter between the hemodialysis sessions in order to address intra luminal sources of infection (Jun Zhang et al., 2016). Antimicrobial solutions (Vancomycin-heparin (2.5mg/ml), Cefazolin-heparin $(5 \mathrm{mg} / \mathrm{ml})$, Gentamicin-Sodium citrate $(40 \mathrm{mg} / \mathrm{ml})$ ) can prevent biofilm formation (Lars Skov Dalgaard et al.,).

Bio film acts as an important source of bacteremia and also a key factor favoring bacterial lodgement and in turn results in bacterial resistance thereby limiting the physician's antibiotic options for therapeutic management of CRBSIs. At the same time, concerns about the toxicity of antimicrobial lock therapy in case of over fill, especially at higher concentrations must be overlooked (Jun Zhang et al., 2016). The risk factors for CRBSIs in chronic hemodialysis patients includes: i) Septicemia, ii) Stenosis, iii) Atheromatous plaques, iv) Aneurysm dilatation, v) Low serum albumin level, vi) Low BMI, vii) Diabetes, viii) infections, ix) High BUN (Tarek et al., 2016). The specific aim of this study is to determine the incidence rate of Catheter Related Blood Stream Infections among chronic hemodialysis patients with central venous catheters in- situ. The objectives of our study are to evaluate the bacteriological profile, antibiotic susceptibility pattern and to screen for specific drug resistance among the culture isolates if any.

A study conducted by Masashi Suzuki et al., on Bacteremia in hemodialysis patients showed that the risk of bacteremia was higher and the common causative organisms resulting in bacteremia were found to be Gram-positive bacteria (Masashi Suzuki et al., 2016). Intricate details of review regarding Intravascular Catheter-Related Blood Stream Infections viz., epidemiology, pathogenesis and microbiology of CRBSIs and current guidelines for the diagnosis, management and prevention of these infections are very well elucidated by Harshal Shah et al., in their study done elsewhere (Harshal Shah et al., 2013). Deborah Friedman et al., developed a new classification scheme for CRBSIs that distinguishes community- acquired CRBSI and nosocomial acquired CRBSI (Friedman $e t$ al., 2002). The most common risk factor for bacteremia in hemodialysis patients is the use of central venous catheters. Previous hospitalization in turn serves as a second leading risk factor for CRBSIs. Membrane reuse was also found to be an important risk 
factor for septicemia. Likewise, Diabetes mellitus and Hypoalbuminemia $(<3.5 \mathrm{mg} / \mathrm{dl})$ were also found to be important risk factor for recurrent bacteremia. Analysis of "United States Renal Data System" reported 50\% higher risk of septicemia in hemodialysis patients who were on temporary catheter. (Abhilash Chandra et al., 2016) Bacteremia may be the result of either extra luminal or intra luminal catheter surface infections.

Extra luminal catheter surface infections are associated with percutaneous catheters. Following catheter hub contamination, infection spreads by exit site infection with subsequent migration into the blood stream along the extra luminal surface. Other unusual causes reported were intrinsic contamination of infusates and hematogenous seeding from distant infections (Harshal Shah et al., 2013).

According to the guidelines for the diagnosis and management of the intravascular catheterrelated infection by the Infectious Diseases Society of America (IDSA), a definitive diagnosis of CRBSI require/s the following: (a) a set of peripheral blood cultures; (b) blood cultures from the peripheral vein and from a culture of the catheter tip; or (c) cultures from an arterial and venous catheter hub that meet differential time-to-positivity criteria (Masashi Suzuki et al., 2016). In this study, blood cultures from the peripheral vein and from a culture of the catheter tip were taken into account for diagnosis. In addition to this, Catheter Related Blood Stream Infections were suspected in a patient with an intravascular catheter who developed the signs of septicemia viz., fever, dyspnoea, edema and were also considered to have CRBSIs. Extensive research data are available which reports Antibiotic lock therapy significantly reduces the incidence of CRBSIs. Commonly used antibiotics for antibiotic lock therapy are Gentamicin, Cefotaxime, Cefazolin, Minocycline and Vancomycin. Generally the treatment of bacteremia includes administration of systemic antibiotics and care of the catheter site (Masashi Suzuki et al., 2016).

The main aim of this study to determine the prevalence of Catheter Related Blood Stream Infections (CRBSIs) among chronic hemodialysis patients with central venous catheters in-situ attending dialysis unit of our hospital.

The objectives of this study include, to evaluate the bacteriological profile of Catheter Related Blood Stream Infections in chronic hemodialysis patients with central venous catheter in- situ. To detect the Antibiotic susceptibility pattern of the clinical isolates identified. And to screen for the specific drug resistance patterns among clinical isolates.

\section{Inclusion criteria}

Chronic kidney disease patients (age $\geq 18$ years) with tunneled central venous catheter in-situ for hemodialysis presenting with/without the signs and symptoms of septicemia.

\section{Exclusion criteria}

Patients who had not given informed written consent.

Patients positive for Hepatitis B, HCV, HIV were excluded.

Patients who had taken antibiotics in the last 1 month.

Chronic kidney disease Patients on nontunneled catheters.

\section{Materials and Methods}

A Cross sectional study was undertaken after obtaining clearance from Institutional Human Ethics Committee (IHEC). Informed written consent was obtained from the study 
participants viz., In-patients and Out-patients clinically diagnosed to have chronic kidney disease from Medicine department constitutes the study population. Blood samples (5ml$10 \mathrm{ml}$ ) were collected from catheter site and also from peripheral vein under sterile and aseptic conditions in a blood culture bottle (BacT/ALERT $^{\mathrm{R}}$ FA plus 410851, Automated Blood Culture System) (Liesa et al., 2016). Second blood sample was collected from the same patient at a time interval of 10-15 minutes (Wasse et al., 2007) and were transported immediately to the Microbiology laboratory. The specimens obtained were processed by performing routine diagnostic tests like Aerobic culture, appropriate biochemical tests to aid in the identification of bacteria. The Antibiotic Susceptibility Testing was done and susceptibility patterns observed, analyzed and recorded as per Standard Operating Procedures.

The blood samples after inoculation were incubated in an automated blood culture system $\left(\mathrm{BacT}_{\text {ALERT }}{ }^{\mathrm{R}}\right.$ ) for a period of 5 days. In the meantime, a beep alert was considered as positive after which the bottles were taken out and Semi-quantitative blood cultures were done with $84 \mu \mathrm{L}$ volume in a disposable $5 \mathrm{ml}$ sterile plastic syringe on routine plating media viz., Blood Agar Plate (BAP), Mac Conkey Agar Plate (MAP), Chocolate Agar Plate (CAP) and were then incubated aerobically at $37^{\circ} \mathrm{C}$ for $24-48$ hours (Dubey et al., 2010). The culture plates were read for presence or absence of bacterial growth. In case of positive cultures, Gram stain was performed and gram reactions were recorded. The culture isolates were identified by routine methods as per standard guidelines by interpretation of colonial morphology, gram reaction and routine Bio-chemical reactions and also with some other special tests like catalase, oxidase, nitrate, motility and etc. Interpretation of "culture sterile" was given as preliminary report after 2 days and as final report after 5 days of incubation in case of no growth.

Antibiotic Susceptibility Test (AST) were done on Mueller-Hinton Agar using by Kirby Bauer's disk diffusion method according to Clinical and Laboratory Standards Institute (CLSI) guidelines with 0.5 and $1.0 \mathrm{Mac}$ Farland turbidity standard of Gram-negative bacteria and Gram-positive bacteria respectively. A lawn culture of test organism was made on Mueller Hinton Agar Plate (MHAP) and commercially available antibiotic discs of fixed antibiotic concentrations were placed individually ( 5 to 6 antibiotic discs were kept at a distance of 1.5 $\mathrm{cm}$ between each disc on standard $90 \mathrm{~mm}$ Petri plate). MHAP was incubated at $37^{\circ} \mathrm{C}$ under aerobic incubation for a period of 16-24 hours and Antibiotic Susceptibility Patterns were noted by reading the zone of inhibition around the antibiotic disc (Sahu et al., 2012). Specific drug resistance patterns viz., MRSA, VRE, CRE, AmpC, ESBL, K1 enzyme production were identified by combination or double disk potentiation method and etc., as per CLSI 2018 guidelines. Quality Control for Culture and AST were done using standard reference strains like Escherichia coli ATCC 25922 and Staphylococcus aureus ATCC 25923 standard reference strains. Data entry and Statistical analysis were done using IBM SPSS 23.0 Statistical Analysis software.

\section{Results and Discussion}

A total of 60 paired blood samples were collected from the study populations during the two months period. The male and female ratio was 1.07:0.93 with slight male preponderance (male 31 out of $60(51.66 \%) \&$ female 29 out of 60 (48.33\%)). Out of the 60 samples processed for culture and sensitivity, nearly 24 samples (40\%) were "Culture Positives" and 36 samples (60\%) were sterile and termed "Culture Negatives" after 5 days of incubation. 
Among culture positives, 19 out of 24 $(79.16 \%)$ were identified as "Gram positive bacteria" namely Methicillin Resistant Coagulase Negative Staphylococcus (MRCONS, 8 out of 24, 33.33\%), Methicillin Sensitive Coagulase Negative Staphylococcus (MSCONS, 3 out of 24, 12.5\%), Methicillin Resistant Staphylococcus aureus (MRSA, 2 out of 24, 8.33\%), Enterococcus (1 out of 24, $4.16 \%$ ), Micrococci (2 out of 24, 8.33\%), Aerobic spore bearing gram positive bacilli ( 3 out of $24,12.5 \%$ ).

About 5 out of $24(20.83 \%)$ culture positive isolates were identified as "Gram negative bacteria" namely Enterobacter species (3 out of 24, 12.5\%), Escherichia coli (2 out of 24, $8.33 \%)$.

The predominant Gram positive bacteria observed in our study was MRCONS followed by MSCONS, MRSA and Micrococci.

The predominant Gram negative bacteria that were isolated from the study population was found to be Enterobacter species followed by Escherichia coli.

MRCONS culture isolates exhibited resistance to Erythromycin and Penicillin G. MSCONS manifested resistance to Erythromycin. MRSA manifested resistance to Gentamicin and Penicillin G. Among Enterococcal isolates, Gentamicin and Doxycycline showed resistance. In our study, all the Gram positive bacteria were found to be susceptible to Vancomycin, Linezolid, Doxycycline and Gentamicin. Among Gram negative isolates Enterobacter species showed resistance to Amoxycillin Clavulanate, Ampicillin and Ciprofloxacin. This isolate was susceptible to Tigecycline, Amikacin, Imipenem and
Tobramycin. Escherichia coli isolates displayed resistance to Ampicillin, Amikacin and were susceptible to Ceftazidime, Cefotaxime, Piperacillin and Tazobactam.

In our study, the prevalence of Catheter Related Blood Stream Infections among chronic hemodialysis patients with central venous catheter in- situ was found to be $40 \%$. Gram positive bacteria comprised $3 / 4^{\text {th }}$ of the cases while the remaining $1 / 4^{\text {th }}$ were Gram negative bacteria.

Most of the studies showed that prevalence rate of bacteremia due to MRSA (Masashi Suzuki et al., 2016) as high whereas in our study prevalence rate of MRCONS $(33.33 \%)$ was higher than that of MRSA $(8.33 \%)$.

Recommended practices for prevention of catheter related bloodstream infections include: a) To limit the insertion of catheter to the trained personnel; b) To use $>0.5 \%$ chlorhexidiene plus alcohol or povidoneiodine for preparation of skin; c) Strict adherence to maximum sterile barrier precautions like head cap, face mask, sterile gown, sterile glove and sterile full body drape while insertion of catheter and also during guidewire exchange; d) Complete hand hygiene prior to insertion, assessment or changing the dressing is mandatory; e) To use subclavian vein instead of internal jugular or femoral vein; f) To scrub catheter hubs with appropriate antiseptics after cap is removed and before accessing; g) To replace gauze dressings every 2 days once and to replace semi-permeable transparent dressing every 7 days once; h) To remove intravascular catheter when it is no longer required for patient care (recommended) (Harshal Shah et al., 2013). 
Fig.1 Management guidelines for CRBSIs in chronic hemodialysis patients on Central Venous Catheter

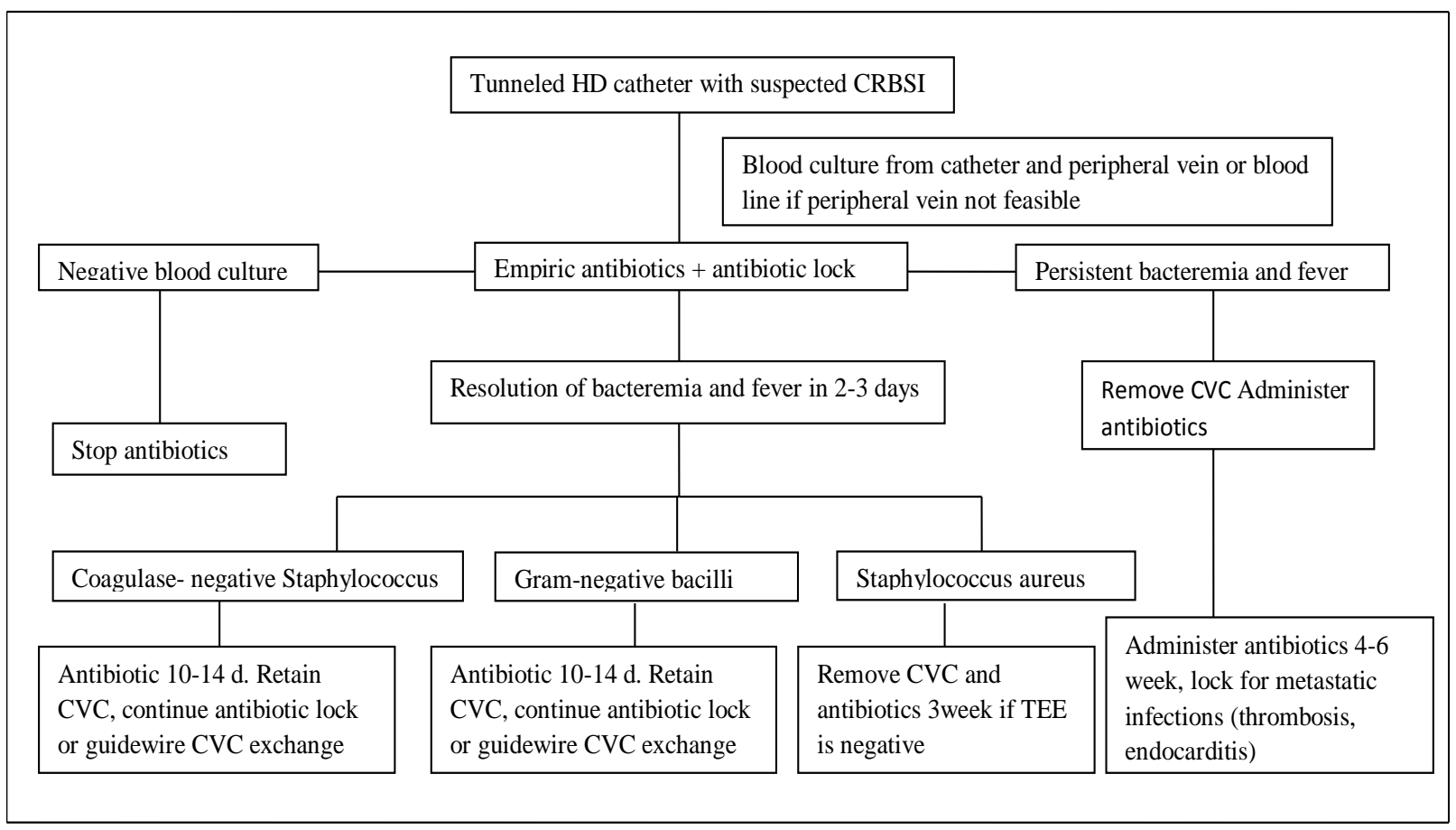

\section{Bacteriological Profile}

MR CONS MS CONS MRSA Enterobacter Enterococcus Micrococci E.coli Gram positive rods

Gram positive rods

3

$(12.5 \%)$

Escherichia coli
2
$(8.33 \%)$

Micrococci

2

$(8.33 \%)$

Enterococcus 1

$(4.16 \%)$

MS CONS
3

(12.5\%)

$\begin{array}{cc}\text { Enterobacter } & \\ 3 & \text { MRSA } \\ (12.5 \%) & 2 \\ & (8.33 \%)\end{array}$


The potential limitations of this study are: a) The Sample size is considerably low to understand the epidemiology of CRBSIs. b) Follow-up of patients was difficult. c) Anaerobic bacteriological profile was not done. d) Antibiotic consumption by patients during the last 1 month could have had been the reason for culture negatives in cases of unreliable drug history given by the patients. e) Lack of hospital antibiotic policy exempted as to correlate right prescription of antibiotics by Physician according to the culture \& sensitivity report given by the Clinical Microbiologist. f) Drug resistant pathogens identified were not subjected for molecular confirmation by PCR and other special confirmatory tests. g) Lack of hospital environment surveillance system to screen for prevailing nosocomial pathogens creates concerns about the understanding of epidemiology of CRBSIs. h) Data about the prevalence of nasal MRSA carrier state among health care providers (doctors, nurses, medical students and sanitary workers) and other specific drug resistance pathogenic bacteria are unavailable in our hospital.

\section{Implications of the study}

The study will give a first-hand research experience to the principal investigator in data collection, sample collection and performing special laboratory tests and co-relating their results with clinical outcome of the patients.

Details like Prevalence of CR-BSI's, Bacteriological profile, antibiogram of susceptible and drug resistant bugs will help understand the nosocomial pathogens causing these infections and in turn would help us revise the Hospital Antibiotic Policy. To Plan and execute periodic Environmental surveillance for Nosocomial pathogens. Designing good preventive measures in the form of practical, low cost, low-technology system that is feasible to implement.
To conclude, when we encounter hemodialysis patients with catheter as vascular access presenting with fever, the first diagnosis to be considered is CRBSIs (Abhilash Chandra et al., 2016). The prevalence of CRBSIs among chronic kidney disease patients receiving hemodialysis was found to be $40 \%$. Evidence- based guidelines is available to plan appropriate antibiotic treatment and holistic catheter management when infection occurs. Risk of CRBSI can be reduced by optimizing catheter insertion and maintenance and by also removing catheters when they are no longer needed. Improving basic infection control measures viz., hand hygiene and other universal safety precautions. Increasing the awareness and educating the health care providers and patients, could significantly reduce the occurrence of bacteremia and could possibly aid in early diagnosis and prompt therapeutic management of CRBSIs. Although this is difficult because the blood streams of these patients frequently exposed to extracorporeal devices (Masashi Suzuki et al., 2016).

\section{Acknowledgment}

This study was done as a part of Indian Council of Medical Research Short Term Studentship project (ICMR STS 2018). I thank ICMR for accepting my research project. I am thankful to the Dean and HOD of Microbiology for permitting to do the study.

\section{References}

Harshal Shah, MBBS ${ }^{1}$, Wendelyn Bosch, $\mathrm{MD}^{1}$, Kristine M. Thomson, $\mathrm{MD}^{2}$, and Walter C. Hellinger, MD ${ }^{1}$. Intravascular Catheter-Related Bloodstream Infection. The Neurohospitalist. 2013; 3(3):144-151.

Friedman ND ${ }^{1}$, Kaye KS, Stout JE, McGarry SA, Trivette SL, Briggs JP, Lamm W, 
Clark C, MacFarquhar J, Walton AL, Reller LB, Sexton DJ. Health care-associated bloodstream infections in adults: a reason to change the accepted definition of community-acquired infections. 2002 Nov19; 137(10): 791-7.

Masashi Suzuki, Nobuhiko Satoh, Motonobu Nakamura, Shoko Horito, George Seki, Kyoji Moriya. Bacteremia in hemodialysis patients, World Journal of Nephrology, 2016; Vol. 6; 5(6): 489496.

Ramasubramanian, V., Vivek Iyer, Sandeep Sewlikar and Anish Desai. Epidemiology of healthcare acquired infection-An Indian perspective on surgical site infection and catheter related blood stream infection. Indian Journal of Basic and Applied Medical Research, 2014; Vol-3, Issue-4, P. 4663.

Abhilash Chandra, Anupam Das, Manodeep Sen, Divya Srivastava. Hemodialysis catheter related blood stream infection in ESRD patients: incidence, outcome and antibiogram of the isolated organisms, International Journal of Advances in Medicine, 2016; Vol-3, Issue-4, P. 912-919.

Jun Zhang, Rong-Ke Li, Kee-HsinChen, Long Ge, Jin-Hui Tian. Antimicrobial local solutions for the prevention of catheterrelated infection in patients undergoing hemodialysis: study protocol for network meta-analysis of randomised controlled trials. BMJ Open 2016; e010264.
Lars Skov Dalgaard, Mette Norgaard, Bente Jespersen, Soren Jensen-Fangel, Lars Jorgen Ostergaard, Henrik Carl Schonheyder, Ole Schmeltz Sogaard. Risk and Prognosis of Bloodstream Infections among Patients on Chronic Hemodialysis: A Population-Based Cohort Study, PloS ONE 10(4): e0124547.

Tarek A. Ghonemy, Salama E. Farag, Sameh A. Soliman, Essam M. Amin, Amal A. Zidan. Vascular access complications and risk factors in haemodialysis patient: A single centre study, Alexandria Journal of Medicine, 2016; Vol-52, Issue-1, P. 67-71.

Liesa M. Miller, Edward Clark, Christiane Dipchand, Swapnil Hiremath, Joanne Kappel, Mercedeh Kiaii, Charmaine Lok, Rick Luscombe, Louise Moist, Matthew Oliver and Jennifer MacRae. Hemodialysis Tunneled CatheterRelated Infections: Canadian Journal of Kidney Health and Disease; 2016: Vol3, P. 1-11.

Wasse, H, Kutner N, Zhang R, Huang Y. Association of initial hemodialysis vascular access with patient-reported health status and quality of life. Clin Journal Am Soc Nephral 2007; Vol-2, P.708-714.

Dubey D, Rath S, Sahu MC, Padhy RS. Culture methods for gram negative bacilli. 2010; 13:766-789.

Sahu MC, Rath S, Dubey D, Debata MK, Padhy. Antibiotic Susceptibility Test to determine resistance of bacilli. 2012; 16: 313-322.

\section{How to cite this article:}

Karthiga, S., A. Dhanasekaran and Someshwaran, R. 2018. Bacteriological Profile and Antibiogram of Catheter Related Blood Stream Infections (CR-BSIs) among Chronic Hemodialysis Patients Attending Tertiary Care Medical College Hospital. Int.J.Curr.Microbiol.App.Sci. 7(11): 1121-1128. doi: https://doi.org/10.20546/ijcmas.2018.711.130 